Acta Crystallographica Section E

Structure Reports

Online

ISSN 1600-5368

\title{
Benzyltrimethylammonium dihydrogen orthophosphate monohydrate
}

The title compound, $\mathrm{C}_{10} \mathrm{H}_{16} \mathrm{~N}^{+} \cdot \mathrm{H}_{2} \mathrm{PO}_{4}{ }^{-} \cdot \mathrm{H}_{2} \mathrm{O}$, crystallizes in the centrosymmetric space group $P 2_{1} / c$ with two independent molecules in the asymmetric unit. Therefore, nonlinear optical properties are absent. The crystal packing is governed by hydrogen bonds, so that the phosphate anions are linked headto-tail, forming chains running parallel to the $a$ direction. These chains in turn are interconnected by hydrogen bonds to water molecules, forming hydrogen-bonded molecular layers stacked parallel to the $a b$ plane.

\section{Comment}

Inorganic salts of phosphoric acids form compounds that exhibit many interesting structural properties, such as phase transitions, physical properties like ferroelectricity, and optical nonlinear phenomena like second harmonic generation, as is the case for potassium dihydrogen orthophosphate (KDP; Rashkovich, 1991). As for organic salts, a general synthetic route to obtain organo dihydrogen phosphate crystals has been detailed in the literature (Masse \& Zyss, 1991). In all these compounds, there is an inorganic hydrogen-bond subnetwork formed by the dihydrogen orthophosphate anions. The organic cation benzyltrimethylammonium (hereafter BTA) is known to form with selenious acid a compound, benzyltrimethylammonium trihydrogen selenite, which exhibits structural phase transitions (de Matos Gomes et al., 1995). No other crystal structures of BTA salts with other acid oxoanions have been reported in the literature. On the other hand, the structures of the related tetramethylammonium (Ohama et al., 1987) and $N$-benzylmethylammonium (Elaoud et al., 1998) dihydrogen orthophosphates have been reported.

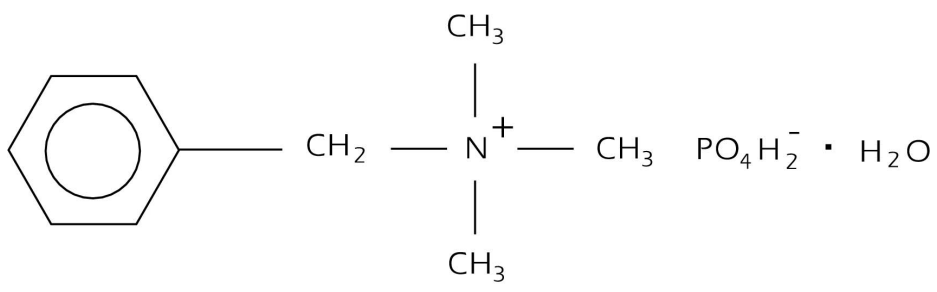

(I)
Received 16 February 2005 Accepted 18 March 2005 Online 25 March 2005
(C) 2005 International Union of Crystallography Printed in Great Britain - all rights reserved

\author{
Key indicators \\ $T=293 \mathrm{~K}$ \\ For details of how these key indicators wer \\ automatically derived from the article, see \\ http://journals.iucr.org/e.
}




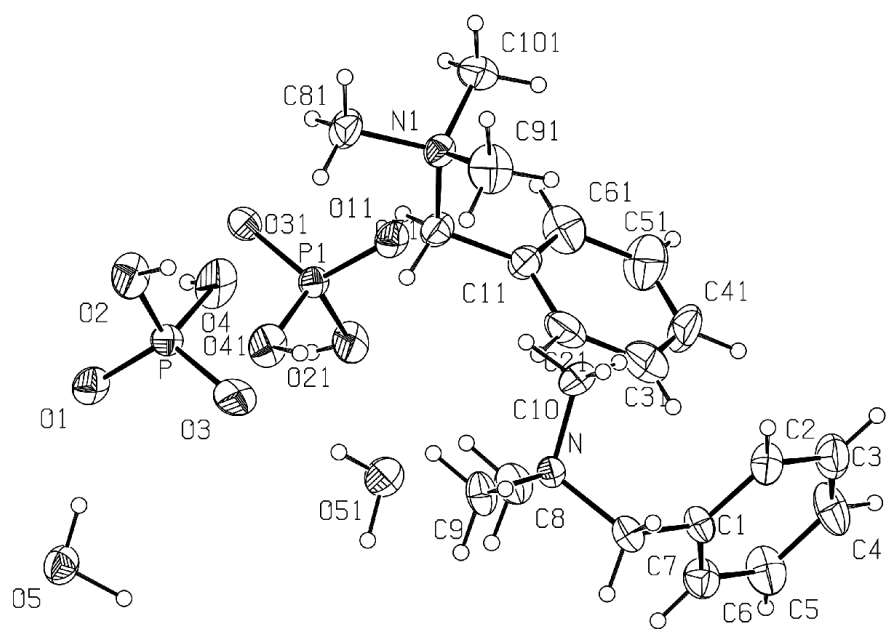

Figure 1

An ORTEPII plot (Johnson, 1976) of (I), showing 30\% probability displacement ellipsoids. $\mathrm{H}$ atoms are shown as spheres of arbitrary radii.

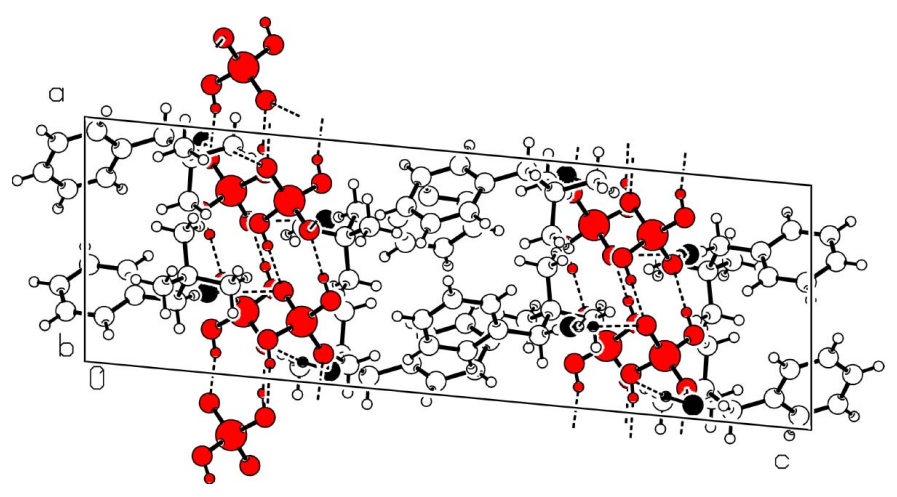

Figure 2

Molecular packing of (I), viewed along the $b$ direction, showing the hydrogen bonds (dashed lines) between phosphate ions.

As may be expected, the two $\mathrm{P}-\mathrm{O}$ distances for the $\mathrm{OH}$ groups are significantly longer than the other two $\mathrm{P}-\mathrm{O}$ distances. The bond lengths in both symmetry-independent BTA cations are very similar and also agree with previous work (de Matos Gomes et al., 1995), whereas the bond angles show a larger range as a result of the intermolecular crystal packing forces. The most interesting crystallographic feature in these organic salts is the hydrogen-bond packing network. As in the case of 1,1,3,3-tetramethylguanidinium dihydrogen orthophosphate (Criado et al., 2000), the phosphate anions are arranged in linear chains (Fig. 2). These chains parallel to the $a$ direction are formed by the two phosphate ions in the asymmetric unit and all the translationally equivalent ions along the $a$ direction. Each ion is connected by two hydrogen bonds to each neighbouring phosphate ion in the chain, so that alternate ions in the chain are related by a lattice translation $a$ and adjacent ions are symmetrically-independent. In addition to this, linear chains related to each other by a screw axis are interconnected by two water molecules, forming four hydrogen bonds (Fig. 3), resulting in a crystal packing of hydrogen-bonded layers stacked parallel to the $a b$ plane so

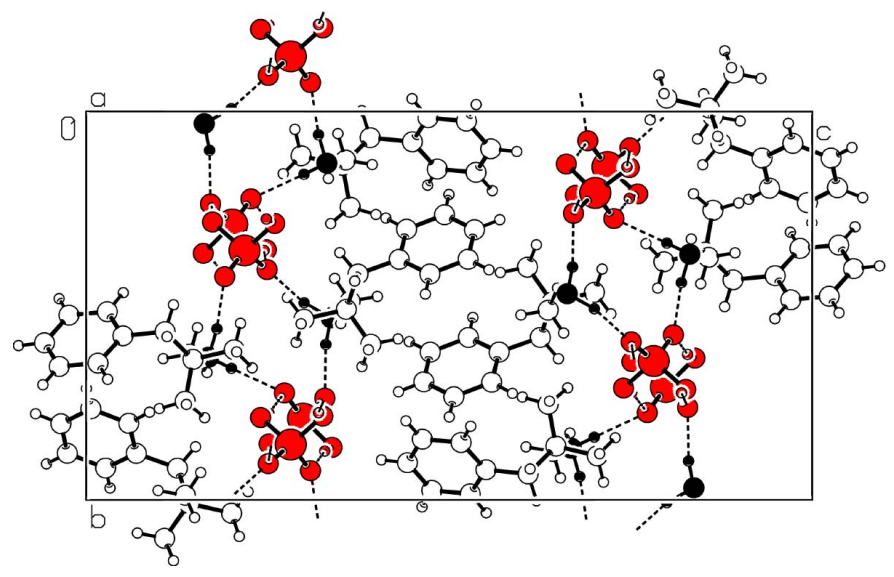

Figure 3

Molecular packing of (I), viewed along the $a$ direction, showing the hydrogen bonds (dashed lines) involving water molecules.

that adjacent layers are related by an inversion centre and alternate layers are separated by a lattice period $c$.

Five $\mathrm{C}-\mathrm{H} \cdots \mathrm{O}$ short contacts not depicted in the Figures have been found.

\section{Experimental}

The present compound was synthesized by mixing equimolar amounts of benzyltrimethylammonium hydroxide and phosphoric acid in a mixed solvent of water and methanol. The crystals were grown by slow evaporation and were of prismatic habit, colourless and transparent.

\section{Crystal data}

$\mathrm{C}_{10} \mathrm{H}_{16} \mathrm{~N}^{+} \cdot \mathrm{H}_{2} \mathrm{PO}_{4}^{-} \cdot \mathrm{H}_{2} \mathrm{O}$

$M_{r}=265.24$

Monoclinic, $P 2_{1} / c$

$a=8.295(2) \AA$

$b=13.178(7) \AA$

$c=24.714(4) \AA$

$\beta=95.412(1)^{\circ}$

$V=2689.5(16) \AA^{3}$

$Z=8$

$D_{x}=1.310 \mathrm{Mg} \mathrm{m}^{-3}$

$D_{m}=1.30 \mathrm{Mg} \mathrm{m}^{-3}$

\section{Data collection}

Enraf-Nonius CAD-4

diffractometer

$\omega-2 \theta$ scans

Absorption correction: none

4723 measured reflections

4723 independent reflections

3482 reflections with $I>2 \sigma(I)$

Refinement

Refinement on $F^{2}$

$R\left[F^{2}>2 \sigma\left(F^{2}\right)\right]=0.051$

$w R\left(F^{2}\right)=0.143$

$S=1.01$

4723 reflections

419 parameters

$\mathrm{H}$ atoms treated by a mixture of independent and constrained refinement
$D_{m}$ measured by flotation in bromobenzene and acetone Mo $K \alpha$ radiation

Cell parameters from 25 reflections

$\theta=7-12^{\circ}$

$\mu=0.21 \mathrm{~mm}^{-1}$

$T=293(2) \mathrm{K}$

Prism, colourless

$0.48 \times 0.40 \times 0.28 \mathrm{~mm}$

$$
\begin{aligned}
& \theta_{\max }=25.0^{\circ} \\
& h=-9 \rightarrow 9 \\
& k=0 \rightarrow 15 \\
& l=0 \rightarrow 29 \\
& 3 \text { standard reflections } \\
& \quad \text { frequency: } 60 \text { min } \\
& \quad \text { intensity decay: none }
\end{aligned}
$$

$$
\begin{aligned}
& w=1 /\left[\sigma^{2}\left(F_{o}^{2}\right)+(0.1215 P)^{2}\right. \\
& \quad+0.1493 P] \\
& \text { where } P=\left(F_{o}^{2}+2 F_{c}^{2}\right) / 3 \\
& (\Delta / \sigma)_{\max }=0.010 \\
& \Delta \rho_{\max }=0.49 \AA^{-3} \\
& \Delta \rho_{\min }=-0.58 \mathrm{e}^{-3}
\end{aligned}
$$


Table 1

Selected geometric parameters $\left(\AA{ }^{\circ}\right)$.

\begin{tabular}{llll}
\hline $\mathrm{P}-\mathrm{O} 1$ & $1.492(2)$ & $\mathrm{P} 1-\mathrm{O} 31$ & $1.494(2)$ \\
$\mathrm{P}-\mathrm{O} 3$ & $1.492(2)$ & $\mathrm{P} 1-\mathrm{O} 11$ & $1.494(2)$ \\
$\mathrm{P}-\mathrm{O} 2$ & $1.556(2)$ & $\mathrm{P} 1-\mathrm{O} 41$ & $1.569(2)$ \\
$\mathrm{P}-\mathrm{O} 4$ & $1.560(2)$ & $\mathrm{P} 1-\mathrm{O} 21$ & $1.571(2)$ \\
$\mathrm{N}-\mathrm{C} 10$ & $1.488(4)$ & $\mathrm{N} 1-\mathrm{C} 91$ & $1.487(4)$ \\
$\mathrm{N}-\mathrm{C} 8$ & $1.490(4)$ & $\mathrm{N} 1-\mathrm{C} 81$ & $1.504(4)$ \\
$\mathrm{N}-\mathrm{C} 9$ & $1.497(4)$ & $\mathrm{N} 1-\mathrm{C} 71$ & $1.522(4)$ \\
$\mathrm{N}-\mathrm{C} 7$ & $1.524(4)$ & & \\
$\mathrm{O} 1-\mathrm{P}-\mathrm{O} 3$ & $116.4(1)$ & $\mathrm{O} 31-\mathrm{P} 1-\mathrm{O} 11$ & $117.9(1)$ \\
$\mathrm{O} 1-\mathrm{P}-\mathrm{O} 2$ & $108.2(1)$ & $\mathrm{O} 31-\mathrm{P} 1-\mathrm{O} 41$ & $107.8(1)$ \\
$\mathrm{O} 3-\mathrm{P}-\mathrm{O} 2$ & $108.6(1)$ & $\mathrm{O} 11-\mathrm{P} 1-\mathrm{O} 41$ & $109.7(1)$ \\
$\mathrm{O} 1-\mathrm{P}-\mathrm{O} 4$ & $109.6(1)$ & $\mathrm{O} 31-\mathrm{P} 1-\mathrm{O} 21$ & $108.2(1)$ \\
$\mathrm{O} 3-\mathrm{P}-\mathrm{O} 4$ & $106.0(2)$ & $\mathrm{O} 11-\mathrm{P} 1-\mathrm{O} 21$ & $106.5(1)$ \\
$\mathrm{O} 2-\mathrm{P}-\mathrm{O} 4$ & $107.7(2)$ & $\mathrm{O} 41-\mathrm{P} 1-\mathrm{O} 21$ & $106.0(2)$ \\
$\mathrm{C} 10-\mathrm{N}-\mathrm{C} 8$ & $109.6(3)$ & $\mathrm{C} 101-\mathrm{N} 1-\mathrm{C} 91$ & $109.3(3)$ \\
$\mathrm{C} 10-\mathrm{N}-\mathrm{C} 9$ & $108.1(3)$ & $\mathrm{C} 101-\mathrm{N} 1-\mathrm{C} 81$ & $108.2(3)$ \\
$\mathrm{C} 8-\mathrm{N}-\mathrm{C} 9$ & $108.2(3)$ & $\mathrm{C} 91-\mathrm{N} 1-\mathrm{C} 81$ & $108.8(3)$ \\
$\mathrm{C} 10-\mathrm{N}-\mathrm{C} 7$ & $111.7(3)$ & $\mathrm{C} 101-\mathrm{N} 1-\mathrm{C} 71$ & $110.5(3)$ \\
$\mathrm{C} 8-\mathrm{N}-\mathrm{C} 7$ & $110.7(2)$ & $\mathrm{C} 91-\mathrm{N} 1-\mathrm{C} 71$ & $112.2(3)$ \\
$\mathrm{C} 9-\mathrm{N}-\mathrm{C} 7$ & $108.4(2)$ & $\mathrm{C} 81-\mathrm{N} 1-\mathrm{C} 71$ & $107.7(2)$ \\
$\mathrm{C} 1-\mathrm{C} 7-\mathrm{N}$ & $113.8(2)$ & $\mathrm{C} 11-\mathrm{C} 71-\mathrm{N} 1$ & $115.6(2)$ \\
\hline
\end{tabular}

Table 2

Hydrogen-bonding geometry $\left(\AA{ }^{\circ}\right)$.

\begin{tabular}{|c|c|c|c|c|}
\hline$D-\mathrm{H} \cdots A$ & $D-\mathrm{H}$ & $\mathrm{H} \cdots A$ & $D \cdots A$ & $D-\mathrm{H} \cdots A$ \\
\hline $\mathrm{O} 5-\mathrm{H} 5 A \cdots \mathrm{O} 1$ & $0.80(5)$ & $2.02(5)$ & $2.807(3)$ & $168(5)$ \\
\hline $\mathrm{O} 5-\mathrm{H} 5 B \cdots \mathrm{O} 11^{\mathrm{i}}$ & $0.86(5)$ & $2.03(5)$ & $2.870(3)$ & $170(4)$ \\
\hline $\mathrm{O} 51-\mathrm{H} 51 A \cdots \mathrm{O} 31$ & $0.79(5)$ & $2.05(5)$ & $2.826(4)$ & $177(5)$ \\
\hline $\mathrm{O} 51-\mathrm{H} 51 B \cdots \mathrm{O}^{\mathrm{ii}}$ & $0.79(5)$ & $2.08(5)$ & $2.860(4)$ & $171(5)$ \\
\hline $\mathrm{O} 2-\mathrm{H} 2 \cdots \mathrm{O} 31$ & 0.82 & 1.82 & $2.629(3)$ & 168 \\
\hline $\mathrm{O} 4-\mathrm{H} 4 \cdots \mathrm{O} 11^{\mathrm{iii}}$ & 0.83 & 1.86 & $2.566(3)$ & 143 \\
\hline $\mathrm{O} 21-\mathrm{H} 21 \cdots \mathrm{O} 3$ & 0.82 & 1.77 & $2.582(3)$ & 170 \\
\hline $\mathrm{O} 41-\mathrm{H} 41 \cdots \mathrm{O} 1^{\text {iv }}$ & 0.82 & 1.79 & $2.579(3)$ & 159 \\
\hline $\mathrm{C} 8-\mathrm{H} 8 B \cdots \mathrm{O} 21^{\mathrm{iii}}$ & $0.90(4)$ & $2.43(5)$ & $3.311(5)$ & $168(4)$ \\
\hline $\mathrm{C} 4-\mathrm{H} 4 A \cdots \mathrm{O} 31^{\mathrm{v}}$ & $0.99(4)$ & $2.41(5)$ & $3.388(4)$ & $173(4)$ \\
\hline $\mathrm{C} 8-\mathrm{H} 8 C \cdots \mathrm{O} 2^{\mathrm{vi}}$ & $0.95(5)$ & $2.53(5)$ & $3.467(5)$ & 169 (4) \\
\hline $\mathrm{C} 81-\mathrm{H} 81 B \cdots \mathrm{O} 3^{\mathrm{ii}}$ & $0.95(5)$ & $2.43(4)$ & $3.372(5)$ & $174(4)$ \\
\hline $\mathrm{C} 71-\mathrm{H} 71 B \cdots \mathrm{O} 5^{\text {vii }}$ & $0.96(4)$ & $2.43(3)$ & $3.359(4)$ & $162(3)$ \\
\hline
\end{tabular}

Symmetry codes: (i) $1-x, y-\frac{1}{2}, \frac{1}{2}-z$; (ii) $1-x, \frac{1}{2}+y, \frac{1}{2}-z$; (iii) $1+x, y, z$; (iv) $x-1, y, z$; (v) $1+x, \frac{1}{2}-y, \frac{1}{2}+z$; (vi) $2-x, y-\frac{1}{2}, \frac{1}{2}-z$; (vii) $2-x, \frac{1}{2}+y, \frac{1}{2}-z$.

C-bound $\mathrm{H}$ atoms were refined freely $[\mathrm{C}-\mathrm{H}=0.79$ (5)-1.06 (5) $\AA$ A]. Water $\mathrm{H}$ atoms were also refined freely. $\mathrm{H}$-atom bond lengths and angles in the phosphate ion were constrained in the refinement [O$\mathrm{H}$ distances given in Table 2; $\left.U_{\text {iso }}(\mathrm{H})=1.5 U_{\text {eq }}(\mathrm{C})\right]$ so that only the torsion angles of the $\mathrm{OH}$ groups have been allowed to refine.

Data collection: CAD-4 Software (Enraf-Nonius, 1989); cell refinement: SET4 (de Boer \& Duissenberg, 1984) and CELDIM (Retting, 1989); data reduction: XRAY76 System (Stewart et al., 1976); program(s) used to solve structure: SIR92 (Altomare et al., 1994); program(s) used to refine structure: SHELXL93 (Sheldrick, 1993); molecular graphics: PLATON (Spek, 2003); software used to prepare material for publication: PARST (Nardelli, 1995) and PARSTCIF (Nardelli, 1991).

This work was supported by the Spanish CICYT project BFM2002-03327 and FEDER funds.

\section{References}

Altomare, A., Cascarano, G., Giacovazzo, C., Guagliardi, A., Burla, M. C., Polidori, G. \& Camalli, M. (1994). J. Appl. Cryst. 27, 435.

Boer, J. L. de \& Duissenberg, A. J. M. (1984). Acta Cryst. A40, C-410.

Criado, A. Diánez, M. J., Pérez-Garrido, S., Fernandes, I. M. L., Belsley, M. \& de Matos Gomes, E. (2000). Acta Cryst. C56, 888-889.

Elaoud, Z., Kamoun, S., Jaud, J. \& Mhiri, T. (1998). J. Chem. Cryst. 28, $313-$ 315.

Enraf-Nonius (1989). CAD-4 Software. Version 5.0. Enraf-Nonius, Delft, The Netherlands.

Johnson, C. K. (1976). ORTEPII. Report ORNL-5138. Oak Ridge National Laboratory, Tennessee, USA.

Masse, R. \& Zyss, J. (1991). Mol. Eng. 1, 141-152.

Matos Gomes, E. de, Matos Beja, A., Paixao, J. A., Alte da Veiga, L., Ramos Silva, R., Martin-Gil, J. \& Martin-Gil, F. J. (1995). Z. Kristallogr. 210, 929933.

Nardelli, M. (1995). J. Appl. Cryst. 28, 659.

Nardelli, M. (1991). PARSTCIF. University of Parma, Italy.

Ohama, N., Machida, M., Nakamura, T. \& Kunifuji, Y. (1987). Acta Cryst. C43, 962-964.

Rashkovich, L. N. (1991). KDP-Family Single Crystals. Bristol: IOP Publishing.

Retting, S. (1989). CELDIM in CAD-4 Software. Version 5.0. Enraf-Nonius, Delft, The Netherlands.

Sheldrick, G. M. (1993). SHELXL93. University of Göttingen, Germany.

Spek, A. L. (2003). J. Appl. Cryst. 36, 7-13.

Stewart, J. M., Machin, P. A., Dickinson, C. W., Ammon, H. L., Heck, H. \& Flack, H. (1976). XRAY76 System. Technical Report TR-446. Computer Science Center, University of Maryland, College Park, Maryland, USA. 